University of Wollongong

Research Online

Faculty of Engineering and Information

Faculty of Engineering and Information

Sciences - Papers: Part A

Sciences

$1-1-2013$

Maximizing the healthcare environment: a systematic review exploring the potential of computer technology to promote self-management of chronic illness in healthcare settings

Mairtin S. McDermott

University of Wollongong, mairtin@uow.edu.au

Alison E. While

Kings College London

Follow this and additional works at: https://ro.uow.edu.au/eispapers

Part of the Engineering Commons, and the Science and Technology Studies Commons

Research Online is the open access institutional repository for the University of Wollongong. For further information contact the UOW Library: research-pubs@uow.edu.au 


\title{
Maximizing the healthcare environment: a systematic review exploring the potential of computer technology to promote self-management of chronic illness in healthcare settings
}

\author{
Abstract \\ Objective The aim of this systematic review is to investigate the effectiveness of using computers to \\ deliver patient self-management programs (PSMPs) to patients with chronic illness in health supported \\ settings. Methods We included randomized controlled trials (RCTs), where the experimental intervention \\ was compared either with an equivalent 'standard' PSMP delivered by staff, usual care or no intervention \\ and reported data either on clinical or behavioral outcomes. We conducted a narrative synthesis, \\ incorporating a small quantitative analysis to enable comparisons across studies. Results A total of 11 \\ studies met the inclusion criteria. There was insufficient evidence to determine whether computer-based \\ PSMPs were superior to standard programs. However, it appeared that these interventions were effective \\ when compared to no intervention. Interventions incorporating behavior change techniques beyond the \\ provision of information appeared more effective than those that did not. Conclusion Evidence from the \\ current review, whilst limited, suggests that computer-based PSMPs, delivered in health-supported \\ settings, show potential for changing health behaviors and improving clinical outcomes in patients with \\ chronic illness. Practice Implications: Although the approach shows promise, it is premature to \\ recommend the integration of these interventions into clinical practice. However, more well designed \\ trials are warranted to test their efficacy and cost-benefit.
}

\section{Keywords}

environment, maximizing, systematic, review, settings, illness, healthcare, chronic, exploring, management, self, promote, technology, computer, potential

\section{Disciplines \\ Engineering I Science and Technology Studies}

\section{Publication Details}

McDermott, M. S. \& While, A. E. (2013). Maximizing the healthcare environment: a systematic review exploring the potential of computer technology to promote self-management of chronic illness in healthcare settings. Patient Education and Counseling, 92 (1), 13-22. 
Title page

Maximizing the healthcare environment: A systematic review exploring the potential of computer technology to promote self-management of chronic illness in healthcare settings.

Máirtín S. McDermott ${ }_{1 *}$ \& Alison E. While 1

${ }_{1}$ Florence Nightingale School of Nursing and Midwifery, King's College London, London, UK

\section{* Corresponding author}

\section{Corresponding author at:}

Florence Nightingale School of Nursing and Midwifery, King's College London, James Clerk Maxwell Building, 57 Waterloo Road, London SE1 8WA.

Telephone: +44 (0) 2078483209

Email: mairtin.mcdermott@gmail.com 


\begin{abstract}
Objective

The aim of this systematic review is to investigate the effectiveness of using computers to deliver patient self-management programs (PSMPs) to patients with chronic illness in health supported settings.
\end{abstract}

\title{
Methods
}

We included randomized controlled trials (RCTs), where the experimental intervention was compared either with an equivalent 'standard' PSMP delivered by staff, usual care or no intervention and reported data either on clinical or behavioral outcomes. We conducted a narrative synthesis, incorporating a small quantitative analysis to enable comparisons across studies.

\section{Results}

A total of 11 studies met the inclusion criteria. There was insufficient evidence to determine whether computer-based PSMPs were superior to standard programs. However, it appeared that these interventions were effective when compared to no intervention. Interventions incorporating behavior change techniques beyond the provision of information appeared more effective than those that did not.

\section{Conclusion}

Evidence from the current review, whilst limited, suggests that computer-based PSMPs, delivered in health-supported settings, show potential for changing health behaviors and improving clinical outcomes in patients with chronic illness. 


\section{Practice Implications}

Although the approach shows promise, it is premature to recommend the integration of these interventions into clinical practice. However, more well designed trials are warranted to test their efficacy and cost-benefit. 


\section{Introduction}

The prevalence of chronic illness is increasing globally, and these conditions are now the primary cause of death and disability in all parts of the world except Africa [1]. In the UK, it is thought that as many as 18 million adults have a chronic illness [2] with the prevalence increasing with age [3]. Similarly, in the USA, almost half of all adults are now living with chronic illness [4], increasing to $92 \%$ of those aged over 65 years [5]. This means that healthcare systems are under increasing pressure to maintain health and promote self-management of conditions, particularly in older people, who are already heavy users of the health service. This pressure is expected to increase in future with reduced national budgets and increased pressure to reduce staffing costs coupled with a growing healthcare workforce crisis [6].

Chronic illnesses, such as diabetes, chronic obstructive pulmonary disorder and coronary heart disease, are caused, maintained or exacerbated by modifiable lifestyle factors, such as, insufficient physical activity, poor nutrition, tobacco use, and excessive alcohol consumption [7]. The health outcomes of these illnesses are also strongly associated with behavior and depend upon good patient self-management. Typically, this involves adherence to treatment (e.g. medication or monitoring regimes) and lifestyle (e.g. diet and exercise) recommendations. For example, people with diabetes need to maintain glycaemic control through diet, exercise and weight control, as well as adherence to therapeutic regimes such as self-monitoring of blood glucose concentrations, foot care, and oral medication or insulin injections [8]. Therefore an important part of patient care is the enhancement of these selfmanagement behaviors [9]. 
The primary mode of facilitating self-management is via patient self-management programs (PSMPs, such as patient education), typically provided by healthcare professionals. Two types of PSMP have been identified: educational interventions which aim to improve self-management by increasing knowledge; and psychological interventions which target emotional, cognitive, and behavioral functioning [8]. However, in the current financial climate, despite the importance of these interventions, healthcare providers are facing increasing resource constraints which restrict the number of staff and amount of time available to deliver these interventions [10]. The use of computers to promote self-management in patients with chronic illness is one potential solution to this challenge [11].

Information and communication technology (ICT) is changing the way that patients interact with the healthcare system, particularly with the development of mobile and web-based interventions [12, 13], but this technology also offers numerous opportunities to maximize the efficiency of the healthcare environment. Patients, particularly those with chronic illness, have to attend for regular medical appointments. However, only $24 \%$ of patients in England are currently seen at their scheduled appointment time [14]. Appropriate integration of computer-based PSMPs into waiting time could minimize the impact of the indirect patient costs (such as work days lost, travelling and waiting time) of appointments, which can in some cases exceed direct costs such as inpatient care, doctor visits and medicines [15], whilst taking advantage of 'teachable moments' around healthcare contacts when patients are primed to receive information on health behaviors [16]. Providing public access computers to deliver PSMPs could also contribute towards bridging the 'digital divide', in particular with older adults who are less likely to own computers or use the 
internet $[17,18]$. Most importantly, from the point of view of the healthcare provider, this technology allows patients access to effective self-management interventions whilst saving healthcare staff time.

A number of other reviews have investigated the use of computers to deliver PSMPs. None, however, have focused on their effectiveness within health-supported settings. Most [10, 19-22] have focused on the effectiveness of all forms of computer-based patient education across settings, with others focusing on their use with specific illnesses [23, 24]. One review investigated the effects of interactive health communication applications for people with chronic illness, but also included online programs, and interventions with a focus beyond patient self-management, including peer and decision support [12]. Similarly, whilst Wofford et al's [25] review was concerned with exploring the potential of using computer-based patient education in the office (clinical) setting, and it also included web- and home-based interventions. The current review, therefore, aims to investigate the effectiveness of using computers to deliver PSMPs to patients with chronic illness in health supported settings. 


\section{Methods}

We followed the Preferred Reporting Items for Systematic Reviews and MetaAnalyses (PRISMA (http://www.prisma-statement.org/) [26]) guidelines throughout the design, conduct and reporting of this systematic review.

\subsection{Selection criteria}

The PICOS (population, intervention, comparison, outcome, study design) approach was used to formulate the research question [27]. We included randomized controlled trials (RCTs), published in English, where the experimental intervention involved delivering a PSMP using a computer (e.g. standalone desktop computer, laptop, computer 'kiosk') in a health-supported setting (i.e. a professional health care setting mediated by health care professionals (e.g. hospital outpatients' department, GPs' surgery, pharmacy), to adults (aged 18 years + ) with a chronic illness for which there is a recommended treatment pathway (e.g. diabetes, heart disease, pulmonary disease, arthritis). A PSMP was defined as any intervention that aims to enable a patient to self-manage their own condition, either through the provision of information or by targeting emotional, cognitive, and behavioral functioning. Although sometimes such programs may also involve family members or other formal or informal caregivers, we were interested in programs targeted solely at the patient. Interventions had to be compared either with usual care (with no self-management element specified), an equivalent 'standard' (i.e. not computer-based) PSMP or no intervention and report the effect of the intervention either on clinical (e.g. HbA1c, mortality etc...) or behavioral (e.g. medication adherence, diet etc...) outcomes. 


\subsection{Study identification}

We searched CENTRAL (The Cochrane Library, Issue 6), EMBASE (via Ovid, 1980 to 2012), INSPEC (ISI Web of Knowledge, 1969 to 2012 and MEDLINE (OVID SP, 1950 to 2012) between May and June 2012. The electronic search strategy was developed by the first author (MSMD), who is experienced in conducting systematic reviews, in consultation with an information specialist. A broad strategy, incorporating keywords sourced from relevant articles together with appropriate subject headings was used to search for studies. The strategy was first developed in MEDLINE before being adapted for use in other databases. Further details can be seen in the review protocol (see additional materials). We also scanned the reference lists of nine systematic reviews covering similar content identified via scoping searches in Google Scholar (using the terms "systematic review", "literature review", “computerized", "patient education" and "health information") [10, 12, 19-25]. We also searched reference lists of included studies. We did not hand search journals.

The first review author (MSMD) pre-screened all titles and abstracts for possible inclusion. The accuracy of the screening process was then checked independently by the second author (AEW) based on a sub-set of included and excluded articles. Those selected were then subject to full-text assessment. Both authors independently assessed the selected articles for inclusion. The first author then extracted data and assessed the quality of each study, the accuracy of which was again checked independently by the second. Any discrepancies were resolved by consensus. 


\subsection{Data extraction}

Details of the intervention and control group populations (e.g. number, demographics and condition), the interventions that they received (intervention content and duration, behavior change techniques used, hardware etc...), and outcomes (measures used, length of follow-up and results) were extracted. The interventions described in the selected studies were complex; covering not only a diverse range of conditions (e.g. diabetes, chronic heart disease etc...), but also a large number of discrete selfmanagement behaviors (e.g. adherence to medication regimen, smoking cessation, healthy eating etc...). In an attempt to manage the complexity of these interventions and inform practice, we sought to determine the 'active ingredients' of each by coding intervention descriptions for behavior-change techniques (BCTs). A BCT is defined as 'any explicit description of intervention content that can alter a participants... behavior, e.g. not including mode or style of delivery' [28] (p316). Taxonomies of BCTs have been created for interventions designed to boost physical activity and healthy eating [29] and for individual interventions for smoking cessation [28] but not, to our knowledge, for computer-based PSMPs. Where possible, we used the BCT terminology described in the above taxonomies with supplementation with techniques specific to computer-based programs designed to facilitating the self-management of chronic illness. Other aspects of delivery that could potentially explain heterogeneity were also noted: the intensity of the intervention (total time of exposure to the computer) and whether the information was tailored to the participants' characteristics.

The methodological quality of each study was assessed according to guidelines in the Cochrane Handbook for Systematic Reviews of Interventions [30] which recommends 
evaluating RCTs in each of the following domains: sequence generation; allocation concealment; blinding of study personnel, data analysts and outcome assessors; incomplete outcome data; selective outcome reporting; other sources of bias (e.g. potential for contamination, suitability of control group in terms of contact/intervention received).

\subsection{Data analysis}

Due to the variation in outcomes and the small number of studies in the review, no meta-analysis was attempted. We conducted a narrative synthesis of studies, incorporating a semi-quantitative analysis to enable comparison of the data across studies. The number of significant findings in each study in favor of the experimental group was noted for each clinical and behavioral outcome and was summarized (i) by study and (ii) by total number of analyses undertaken. Individual studies could contribute multiple findings for each outcome. To reduce the likelihood of a Type I error, where multiple follow-up time points were reported, only the results from the longest follow-up were included in the review. A significance level of $\mathrm{p}<0.05$ was used. 


\section{Results}

\subsection{Selection of studies}

The electronic search strategy retrieved 13,352 unique records. A further seven were identified from the reference lists of related systematic reviews $(n=9,[10,12,19-25])$. In total 13,359 titles and abstracts were screened, of which 304 were retrieved for indepth examination. A total of 11 studies (from 15 articles) met the inclusion criteria. Studies by Glasgow et al [31-33] and Huss et al [34-36] were reported in multiple articles, the most recent of which was utilized in this review. Full details of the screening process can be seen in the PRISMA flow chart (Figure 1).

\section{INSERT FIGURE 1 HERE}

\subsection{Description of the studies}

The study sample sizes ranged from 34 to 476 (total $n=1506$, mean $=137$ ). Mean age ranged from 18.3 to 70.3 years $($ mean $=54.0)$ in the experimental groups and 18.4 to 70.8 years $($ mean $=53.8)$ in the control groups. The samples comprised patients with type I or II diabetes [31, 37, 38], asthma [36, 39, 40], heart failure [41, 42], HIV [43], transient ischemic attack (TIA) or minor stroke [44], and rheumatoid arthritis [45]. Seven studies reported participants' level of education; the proportion of participants with an undergraduate qualification or higher ranged from $25 \%$ to $81 \%$ (mean $=$ $50 \%)$. Only four studies reported ethnicity $[36,37,39,43]$. The majority of studies were conducted in the USA $(n=6)$ with the remainder conducted in Sweden $(n=3)$, Australia $(\mathrm{n}=1)$ and the Netherlands $(\mathrm{n}=1)$. 
Computer-based PSMPs were compared with non-computerized standard PSMPs alone, with usual care or no intervention, or in combination with standard PSMPs compared with standard PSMPs alone or in addition to a non-computerized control. Standard PSMPs typically involved health information delivered by participants' physician or specialist educators, dieticians, or nurses, face-to-face, using audiovisual aids or written materials, either individually or in groups. Standard PSMPs involved primarily the provision of information but occasionally included more complex BCTs such as providing opportunities for social comparison, barrier identification and creating action plans. Usual care typically involved participants' standard treatment plan (e.g. physician visits, check-ups etc...) where no additional educational or behavioral treatment was specified. Outcomes measured varied (see Table 1). Four studies [31, 36, 37, 44] collected data on both behavioral and clinical outcomes, four $[39,42,43,45]$ on behavioral outcomes only and three [38, 40, 41] on clinical outcomes only. Six studies [36-39, 44, 45] reported short-term $(<6$ months) followup, 3 [41-43] reported medium-term follow-up (6 months $-<12$ months) and 2 [32, 40] studies reported long-term follow-up (12 months + ).

\subsection{Description of the interventions}

The majority of studies ( $n=9),[36-42,44,45]$ described themselves as computeraided patient education or instruction, one [43] as computer-based counseling for risky behaviors and one [31] as a computer-assisted intervention providing feedback on barriers to dietary self-management. The experimental intervention in most studies $(n=6)$ comprised of just one session, to a maximum of four sessions ( $n=3$ studies). Length of overall exposure ranged from 22 minutes to 240 minutes (mean $=66$ minutes). 
The majority of studies [31, 36, 37, 39, 40, 43, 45] described interventions that used BCTs beyond the provision of information on their condition, appropriate medication and self-management. Only four studies [31, 37, 43, 44] tailored content of the experimental intervention to participant characteristics. Six studies used a PC, laptop or other standard computer equipment to deliver the intervention [36, 38, 39, 43-45], with three using a computer with a touchscreen [31,37, 42], one a TV set connected to a photo CD player [41] and no equipment details were specified in one study [40]. Six studies attempted to integrate the intervention (or at least part of the intervention) into standard clinical practice, e.g. during waiting time for other appointments, or immediately after a physician appointment [31, 37, 41-44]. Where specified, staff involvement was minimal, amounting to an orientation to the computer program or occasionally being available to assist during the intervention with the practical aspects of using the computer, but without providing any supplementary health information.

\section{INSERT TABLE 1 HERE}

\subsection{Effects of the interventions}

Only one study [38] compared a computer-based PSMP with a standard PSMP. Patients with diabetes randomized to the experimental group viewed a computer-aided learning program across multiple one-hour sessions to cover 16 lessons on diabetes and its management. Analyses revealed that those receiving computer-aided learning had significant improvements in glycosylated haemoglobin (GHb/ HbA1c) levels at three months compared to controls. 
Five studies [31, 39, 40, 43, 45] compared computer-based PSMPs with a control intervention where no patient self-management element was specified (e.g. no intervention, usual care, or assessment only). Each found the experimental intervention to have a beneficial effect on either a behavioral or clinical outcome. One study reported a significant effect on both: all participants in Glasgow et al's study [31] completed a computerized assessment, following which the experimental group participants received a multi-modal intervention aimed at improving the dietary habits of patients with diabetes including an additional dietary barriers assessment, a 20minute goal setting and problem solving session and a computer-based 30-minute interactive video. Participants in the experimental group reported significant reductions in fat-related dietary habits, calories consumed per day, calories consumed from fat and saturated fat at 12 months. Significant reductions in serum cholesterol were also seen in the experimental group, but there were no differences in either Body Mass Index (BMI) or HbA1c.

The computer program tested by Sundberg et al [40] had a significant effect on clinical outcomes when compared to usual care at 12 months. Forced Exhaled Volume in $1 \mathrm{~s}$ (the volume of air that can forcibly exhaled in one second, $\mathrm{FEV}_{1}$ ) improved significantly in young adults (aged 18-25 years) with asthma exposed to the experimental intervention compared to controls, but there were no changes in Forced Vital Capacity (the volume of air that can forcibly exhaled after full inspiration, FVC). There were no significant differences between the groups in the prevalence of respiratory or asthma symptoms. 
The experimental interventions described in the remaining three studies impacted significantly on behavioral outcomes. Gilbert et al [43] compared a computer-based risk assessment plus an interactive computer-based risk-behavior reduction program with a computer-based risk assessment in patients with HIV. The trial reported a reduced risk of drug use and unprotected sex in the experimental compared to the control group but no difference in risky drinking between the groups at six months. Wetstone et al [45] randomized patients with rheumatoid arthritis (RA) to receive either a number of sessions of computer-based education or usual care. Experimental group participants were more likely to report taking increased care in joint protection and reported changes in the amount of time resting (although time resting was reported as both increased and decreased). There was no difference in patient adherence between groups. In Navarre et al's study [39], experimental group participants with asthma or COPD viewed a computer-assisted inhaler technique tutorial on a single occasion and scored significantly higher than a waiting-list control group on observed inhaler technique immediately post-intervention.

The remaining five studies [36, 37, 41, 42, 44] compared computer-based PSMPs combined with a standard PSMP with standard PSMPs alone. Only one study reported a clear beneficial impact of the experimental intervention. Huss et al [36] compared the effects of adding a single session using an interactive computer program to educate adult atopic asthmatics on house dust mite avoidance, to a single session of individual standard education by participants' physicians by comparing it with standard education alone. According to participant self-report and objective assessments, the experimental group implemented significantly more allergen avoidance measures than controls at 12 weeks and also recorded a significant decline 
in mite-allergen levels in the home. The inhaled bronchodilator use per day of the experimental group also significantly decreased below that of the conventional instructed group by 12 weeks. There was no significant difference between groups in observed allergen levels in living room carpets or sofas, symptomatic improvement or mean FEV levels.

Khan et al [37] randomized participants with type II diabetes either to an experimental condition of group-based standard education plus a single session of computer-based learning to standard education plus a read through of a brochure. Both interventions concerned the self-management of diabetes, and each was of benefit to participants. Appropriate use of medication, measured by the number of oral diabetes medications prescribed over three months, improved in experimental versus control participants, but self-reported exercise increased in the control group compared to the experimental group. There was a borderline non-significant $(\mathrm{p}=0.06)$ reduction in $\mathrm{HbA1c}$ in the experimental group compared to controls but no significant differences in diabetes knowledge, diabetes self-efficacy, medication adherence, home glucose monitoring, diet, diabetes foot care, blood pressure, number of blood pressure medications used or insulin use.

The remaining three studies found no differences between groups on either clinical or behavioral outcomes. Linné and Liedholm [41] randomized older patients with heart failure either to standard education plus a single session using an interactive computer program on heart failure, its symptoms and treatment or standard education alone. There was no significant difference between the two groups in mortality and readmission. Those in the intervention group scored significantly higher on 
knowledge than the control group, although only $37 \%$ of patients who finished the study completed the questionnaire. Maasland et al [44] compared standard education delivered to patients with transient ischemic attack (TIA) or minor ischemic stroke plus a single session using an interactive multimedia computer program with standard education alone. The computer program provided information on TIA or stroke, details of medication and tailored information on four personal risk factors for TIA (e.g. level of exercise or smoking). There were no differences between the two groups in blood pressure, serum cholesterol, serum triglyceride, serum low-density lipoprotein (LDL), BMI, number of cigarettes smoked or number of alcoholic drinks consumed or knowledge at 12 weeks. Stromberg et al [42] randomized patients with heart failure either to receive standard education plus a single session using an interactive multimedia program covering information on heart failure and its treatment or standard education alone. There was no significant difference in adherence at six months. The experimental group had a significantly larger increase in knowledge than the control group at six months. There were no differences between the groups in quality of life.

Taken together, these results suggest that computer-aided patient self-management programs for patients with chronic illness are more effective when compared to no intervention or a control with no patient self-management element specified than when compared to standard patient self-management programs alone in combination with standard patient self-management programs for both behavioral (100\% vs. $60 \%$ of studies, $77 \%$ vs. $25 \%$ of analyses) and clinical (100\% vs. $50 \%$ of studies, $33 \%$ vs. $17 \%$ of analyses) outcomes (see Table 2). 
Similarly, the interventions with 'complex' BCTs appeared to be more effective than those with basic techniques, high intensity interventions appeared to be more effective than low intensity interventions and computer-based patient self-management programs appeared to be less effective than non-computer-based approaches in older adults ( $>60$ years). However, no clear pattern emerges when the findings are examined in relation to the tailoring of content (see Table 2).

\section{INSERT TABLE 2 HERE}

\subsubsection{Acceptability of the interventions}

Only four studies reported on the acceptability of the computer-based PSMP. Gilbert et al [43] used a four-item acceptability interview and reported that the majority of participants (97\%) "liked" the program or "liked [it] very much," and 93\% found that it was easy to use. By contrast, $13 \%$ stated that the program was 'too long'. Similarly, $90.4 \%$ of Navarre et al's [39] participants reported that the computer-based tutorial to be either very easy or easy to use, with $92.5 \%$ reporting that the amount of time required to complete the tutorial to be either short or just right, $71.4 \%$ rating the level of inhaler technique explanation as easy and $81.4 \%$ rating the understanding of the material as very easy to easy. Wetstone et al [45] reported that the majority of patients who completed the lesson found it a positive experience, with most reporting that they had learned from the lesson and almost all recommending use of the lesson to other patients, friends and family. In Glasgow et al's study [31], participants receiving the experimental intervention reported significantly higher overall patient satisfaction with their office visit than the control group participants $(p<.002)$. Although it was only assessed in four of the reviewed studies, there was no evidence 
of high attrition in the current review (mean $=9 \%$, range $=0 \%-23 \%$ ) which may be taken as a proxy measure of intervention acceptability.

\subsubsection{Cost of the interventions}

Only one study reported the cost of implementing the intervention [31]. Costs for the intervention as delivered in the study totaled US $\$ 137$ per participant or $\$ 14,755$ in total (including $\$ 2650$ for computer hardware and software). The cost per unit improvement in each outcome was calculated as $\$ 62$ per reduction of each percent of dietary fat, $\$ 105$ per percent reduction in saturated fat and $\$ 8$ per $\mathrm{mg} / \mathrm{dl}$ reduction in serum cholesterol.

\subsection{Methodological quality of the studies}

Two studies $[38,43]$ were judged to have a low risk of bias in all six domains. One study [41] had low risk of bias in five domains, five [36, 37, 39, 40, 44] in four domains, two [31, 45] in three domains and one study [38] was judged to have low risk of bias in only two domains. All studies were judged to have low risk of bias in selective reporting of outcome data, whereas blinding of outcome data was the least frequently met, with only five out of 11 studies judged to have low risk of bias in this domain (see Table 3).

INSERT TABLE 3 HERE 


\section{Discussion and conclusion}

\subsection{Discussion}

To our knowledge, this is the first study to review the effectiveness of computer-based PSMPs delivered in health-supported settings. The evidence, whilst limited, is promising, and suggests that these interventions have the potential to change both health behaviors and clinical outcomes in patients with chronic illness. In the current review, standard PSMPs were provided by health intermediaries including doctors and nurses in health-supported settings. Whilst these approaches offer the advantage of exploiting 'teachable moments', they are cost-intensive as, for the most part, one health professional consultation informs only one person at a time. In the reviewed interventions staff involvement was minimal, and typically involved a brief orientation to operating the computer program. Switching modes of delivery could save health care providers significant costs, whilst simultaneously saving indirect costs for the patient and maximizing the efficiency of their time spent in the health care environment.

There was insufficient evidence, however, to determine whether computer-based PSMPs are superior, or at least equivalent to those delivered by health care professionals as this was addressed in only one study [38], which was of poor quality. There was evidence, however, that these interventions were effective when compared to 'usual care' where no specific patient self-management content was specified, and two previous reviews have reported that computer-based patient education programs were superior to standard education delivered by staff in producing knowledge and clinical gains [10, 21] although this was not found by a third review [25]. 
It has been suggested that these approaches are potentially problematic as the absence of human contact could lead to increased levels of disengagement and attrition [46]. There was no evidence found for any adverse effects of switching to computer-based PSMPs in the current review. Computer-based programs may even be preferred by many patients because they allow participants to move through the intervention at their own pace, so that they are likely to be of benefit for those even with low education level [23] and may be perceived as non-judgemental, particularly if addressing sensitive topics [20]. Although one might consider the range of attributes offered by a combination of standard and computer-based approaches to be optimal, no evidence was found in this review.

Numerous questions remain unanswered. In particular, whether these interventions are effective in older adults. Studies where experimental group participants had a mean age above 60 years appeared to be less effective than those with younger participants. This may be due to only one of these interventions explicitly incorporating design features for older adults [42]. The use of computer technology is fraught with difficulties from the perspective of the older person, with many older people being reticent to use them for a variety of reasons, including issues related to hardware such as screen text being too small and the contrast poor, and the physical manipulations required to control a computer challenging their dexterity [48-50]. Software issues also create difficulties, such as the logic of the interfaces being difficult to understand and use, difficulties searching the internet and issues with low health literacy making computerbased health information difficult to comprehend [49, 50]. This lack of adaptation for older adults is reflective of a general failure of these interventions to maximize the potential of using new technology, as has been noted elsewhere [46]. For example, computer-based approaches should, in theory, allow for increased effectiveness 
through high levels of tailoring and personalization of content. However, only four studies designed their interventions in this way. Despite this review being the first to list BCTs used in PSMPs, it identified few that had not been previously recorded in the taxonomies for interventions to promote physical activity and healthy eating [29] or smoking cessation [28]. As might be expected, newly identified BCTs were linked with the interventions of interest, and primarily concerned the provision of information regarding the patient's condition and the self-management of that condition.

This review had a number of important limitations relating to both the quality of the included studies and the methodology of the review. Restricting the review to published, peer-reviewed research leaves open the possibility that the positive effects of these interventions may be exaggerated by publication bias. Every effort was made, however, to conduct as comprehensive a review as possible, screening over 13,000 titles from four major databases and nine systematic reviews, as well as searching the reference lists of included articles. Most of the included studies were not of high quality, with only two meeting all of the quality criteria. The results must therefore be interpreted with caution. Further limitations include the lack of a meta-analysis to accurately determine the effects of the interventions which would have allowed for a more thorough examination of the effectiveness of these interventions, as well as the effects of any potential moderators. The interventions included in this review were also heterogeneous in terms of information presented, mode and duration, targeted a number of self-management behaviors for a number of different conditions and examined their impact on a diverse range of outcomes. Extracting the BCTs used in 
these interventions, however, allowed us to identify common features that are associated with greater effectiveness.

\subsection{Conclusion}

The primary conclusion of the systematic review is that computer-based PSMPs show potential for changing health behaviors and improving clinical outcomes in patients with chronic illness. However, the review also shows up several important future avenues for research that are essential to fully examine the extent to which these interventions are likely to be of use. First, more well designed trials are needed to test their efficacy, in particular head-to-head comparisons with similar interventions delivered by healthcare professionals which was examined in only one study in the current review. Second, it is imperative that these studies collect data to allow comparison of cost benefit, which was also reported in only one study reviewed here [31]. Third, interventions should incorporate more design features matched to their target population in order to boost their effectiveness. This may be particularly relevant for older adults for the reasons mentioned above, but in general is reflective of a failure to harness the potential of these technologies to communicate effectively with their target audience, including in accurate tailoring and personalization of content. Accompanying this with an exploration of the acceptability of computer technology interventions to both older people and healthcare professionals would be useful in identifying potential implementation issues for their widespread use. Given the current review contained only a subset of the available studies describing PSMPs, further work is needed to list the full range of BCTs used in these interventions. The similarity of BCTs used in these diverse interventions, however, suggests the presence 
of a relatively limited set of BCTs that are broadly applicable and effective across a range of health behaviors and conditions.

\subsection{Practice implications}

Given the results of this review, it would be premature to recommend the use of computer-based PSMPs in clinical settings to promote self-management in patients with chronic illness. It is presently unclear whether these interventions can be used effectively to replace staff time, and in particular whether these interventions can be effective in older adults. It is apparent, however, that educational interventions, i.e. those programs that simply provided information regarding the chronic illness, appropriate medication and self-management, were less effective and should be avoided in favor of psychological interventions that incorporate additional BCTs such as motivational interviewing, barrier identification and modeling of behavior. This is in keeping with previous research which shows that simply providing information is insufficient to produce behavioral change [9]. 


\section{References}

[1] World Health Organisation. Global Status Report on Noncommunicable Diseases 2010. World Health Organization; 2011.

[2] The NHS Information Centre. Quality and Outcomes Framework Achievement Data 2010/11. London: The Health and Social Care Information Centre; 2011.

[3] Office for National Statistics. General Lifestyle Survey Overview: A report on the 2010 General Lifestyle Survey. London: National Statistics; 2012.

[4] Anderson G. Chronic Care: Making the Case for Ongoing Care. New Jersey: Robert Wood Johnson Foundation; 2010.

[5] Hung WW, Ross JS, Boockvar KS, Siu AL. Recent trends in chronic disease, impairment and disability among older adults in the United States. BMC Geriatr. 2011;11:47.

[6] Royal College of Physicians. Hospitals on the Edge: time for action. London: Royal College of Physicians; 2012.

[7] Centers for Disease Control and Prevention. Chronic Diseases and Health Promotion. 2012. Retrieved 02/11/12, from http://www.cdc.gov/chronicdisease/overview/index.htm.

[8] Ismail K, Winkley K, Rabe-Hesketh S. Systematic review and meta-analysis of randomised controlled trials of psychological interventions to improve glycaemic control in patients with type 2 diabetes. Lancet. 2004;363:1589-97.

[9] Wu L, Forbes A, Griffiths P, Milligan P, While A. Telephone follow-up to improve glycaemic control in patients with Type 2 diabetes: systematic review and meta-analysis of controlled trials. Diabet Med. 2010;27:1217-25.

[10] Fox MP. A systematic review of the literature reporting on studies that examined the impact of interactive, computer-based patient education programs. Patient Educ Couns. 2009;77:6-13.

[11] While A, Dewsbury G. Nursing and information and communication technology (ICT): a discussion of trends and future directions. Int J Nurs Stud. 2011;48:1302-10.

[12] Murray E, Burns J, See TS, Lai R, Nazareth I. Interactive Health Communication Applications for people with chronic disease. Cochrane Db Syst Rev. 2005;

19:CD004274.

[13] Free C, Phillips G, Watson L, Galli L, Felix L, Edwards P, et al. The effectiveness of mobile-health technologies to improve health care service delivery processes: A systematic review and meta-analysis. PLoS Medicine. 2013;10:e1001363. 
[14] Care Quality Commission. National Summary of the Outpatients Department 2011 Survey. Quality Care Commission. Retrieved 02/11/12, from http://www.cqc.org.uk/sites/default/files/media/documents/national_summary_op11_ $\underline{0 . p d f}$.

[15] Bahadori K, Doyle-Waters MM, Marra C, Lynd L, Alasaly K, Swiston J, et al. Economic burden of asthma: a systematic review. BMC Pulm Med. 2009;9:24.

[16] Stevens VJ, Glasgow RE, Toobert DJ, Karanja N, Smith KS. One-year results from a brief, computer-assisted intervention to decrease consumption of fat and increase consumption of fruits and vegetables. Prev Med. 2003;36:594-600.

[17] Hardiker NR, Grant MJ. Factors that influence public engagement with eHealth: A literature review. International journal of medical informatics. 2011;80:1-12.

[18] OFCOM. Chapter 4: Internet and web-based content. The Communications Market 2012. 2012.

[19] Jones R. The Role of Health Kiosks in 2009: Literature and Informant Review. Int. J. Environ. Res. Publ. Health. 2009;6:1818-55.

[20] Krishna S, Balas EA, Spencer DC, Griffin JZ, Boren SA. Clinical trials of interactive computerized patient education: implications for family practice. J Fam Practice. 1997;45:25-33.

[21] Lewis D. Computer-based approaches to patient education: a review of the literature. J Am Med Inform Assn. 1999;6:272-82.

26

[22] Lewis D. Computers in patient education. CIN-Comput Inform Nu. 2003;21:8896.

[23] Beranova E, Sykes C. A systematic review of computer-based softwares for educating patients with coronary heart disease. Patient Educ Couns. 2007;66:21-8.

[24] Saksena A. Computer-based education for patients with hypertension: A systematic review. Health Educ J. 2010;69:236-45.

[25] Wofford JL, Smith ED, Miller DP. The multimedia computer for office-based patient education: a systematic review. Patient Educ Couns. 2005;59:148-57.

[26] Moher D, Liberati A, Tetzlaff J, Altman DG. Preferred reporting items for systematic reviews and meta-analyses: the PRISMA statement. PLoS Med. 2009;6:e1000097.

[27] Moher D, Tricco AC. Issues related to the conduct of systematic reviews: a focus on the nutrition field. Am J Clin Nutr. 2008;88:1191-9. 
[28] Michie S, Hyder N, Walia A, West R. Development of a taxonomy of behaviour change techniques used in individual behavioural support for smoking cessation.

Addict Behav. 2011;36:315-9.

[29] Abraham C, Michie S. A Taxonomy of Behavior Change Techniques Used in Interventions. Health Psychol. 2008;27:389-7.

[30] Higgins JPT, Green S. Cochrane Handbook for Systematic Reviews of Interventions Version 5.1.0 (updated March 2011). Available from www.cochranehandbook.

[31] Glasgow RE, La Chance PA, Toobert DJ, Brown J, Hampson SE, Riddle MC. Long-term effects and costs of brief behavioural dietary intervention for patients with diabetes delivered from the medical office. Patient Educ Couns. 1997;32:175-84. 27

[32] Glasgow RE, Toobert DJ, Hampson SE. Effects of a brief office-based intervention to facilitate diabetes dietary self-management. Diabetes Care. 1996;19:835-42.

[33] Glasgow RE, Toobert DJ, Hampson SE, Noell JW. A brief office-based intervention to facilitate diabetes dietary self-management. Health Educ Res. 1995;10:467-78.

[34] Huss K, Huss RW, Squire EN, Carpenter GB, Smith LJ, Salata K, et al. Computer education for asthmatics: what effects? J Nurs Care Qual. 1992;6:57-66.

[35] Huss K, Salerno M, Huss RW. Computer-assisted reinforcement of instruction: effects on adherence in adult atopic asthmatics. Res Nurs Health. 1991;14:259-67.

[36] Huss K, Squire EN, Jr., Carpenter GB, Smith LJ, Huss RW, Salata K, et al. Effective education of adults with asthma who are allergic to dust mites. J Allergy Clin Immun. 1992;89:836-43.

[37] Khan MA, Shah S, Grudzien A, Onyejekwe N, Banskota P, Karim S, et al. A diabetes education multimedia program in the waiting room setting. Diabetes Ther. 2011;2:178-88.

[38] Lo R, Lo B, Wells E, Chard M, Hathaway J. The development and evaluation of a computer-aided diabetes education program. Aust J Adv Nurs. 1996;13:19-27.

[39] Navarre M, Patel H, Johnson CE, Durance A, McMorris M, Bria W, et al. Influence of an interactive computer-based inhaler technique tutorial on patient knowledge and inhaler technique. Ann Pharmacother. 2007;41:216-21.

[40] Sundberg R, Tunsater A, Palmqvist M, Ellbjar S, Lowhagen O, Toren K. A randomized controlled study of a computerized limited education program among young adults with asthma. Resp Med. 2005;99:321-8. 
[41] Linne AB, Liedholm H. Effects of an interactive CD-program on 6 months readmission rate in patients with heart failure - a randomised, controlled trial [NCT00311194]. BMC Cardiovasc Disord. 2006;6:30.

[42] Stromberg A, Dahlstrom U, Fridlund B. Computer-based education for patients with chronic heart failure. A randomised, controlled, multicentre trial of the effects on knowledge, compliance and quality of life. Patient Educ Couns. 2006;64:128-35.

[43] Gilbert P, Ciccarone D, Gansky SA, Bangsberg DR, Clanon K, McPhee SJ, et al. Interactive "Video Doctor" counseling reduces drug and sexual risk behaviors among HIV-positive patients in diverse outpatient settings. PLoS ONE. 2008;3:e1988.

[44] Maasland E, Koudstaal PJ, Habbema JDF, Dippel DWJ. Effects of an individualized multimedia computer program for health education in patients with a recent minor stroke or transient ischemic attack - a randomized controlled trial. Acta Neurol Scand. 2007;115:41-8.

[45] Wetstone SL, Sheehan TJ, Votaw RG, Peterson MG, Rothfield N. Evaluation of a computer based education lesson for patients with rheumatoid arthritis. J

Rheumatol. 1985;12:907-12.

[46] Rosser BA, Vowles KE, Keogh E, Eccleston C, Mountain GA.

Technologicallyassisted

behaviour change: a systematic review of studies of novel technologies for the management of chronic illness. J Telemed Telecare. 2009;15:327-38.

[47] Office for National Statistics. Topic Guide to Older People. 2011. Retrieved 06/11/12, from http://www.statistics.gov.uk/hub/population/ageing/older-people.

[48] Lunn D, Harper S. Providing assistance to older users of dynamic web content. Comput Hum Behav. 2011;27:2098-107.

[49] Consumer Expert Group. Consumer Expert Group report into the use of the Internet by disabled people: barriers and solutions 2009. Retrieved 06/11/12, from http://www.dundeecity.gov.uk/dundeecity/uploaded_publications/publication_1679.p df

[50] Becker SA. A study of web usability for older adults seeking online health resources. ACM T Comput-Hum Int. 2004;11:388-406. 